\title{
Characterization of Staphylococcal Cassette Chromosome mec (SCCmec) in Methicillin-Resistant Staphylococcus epidermidis Strains Isolated from Biomaterial-Associated Infections and their Antibiotic Resistance Patterns
}

\author{
EWA SZCZUKA ${ }^{1 *}$, KAROLINA BOSACKA² and ADAM KAZNOWSKI ${ }^{1}$ \\ ${ }^{1}$ Department of Microbiology, Institute of Experimental Biology, Faculty of Biology, \\ Adam Mickiewicz University, Poznań, Poland \\ ${ }^{2}$ Department of Microbiological and Laboratory Diagnostics, Bacteriological Laboratory, \\ Regional Hospital in Poznań, Poznań, Poland
}

Submitted 10 March 2015, revised 12 June 2015, accepted 30 June 2015

\begin{abstract}
This work aims to provide an insight into staphylococcal cassette chromosome mec elements and antibiotic resistance in clinical isolates of Staphylococcus epidermidis. The dominating type was SCCmec - IV. Fifteen isolates were assigned to SCCmec type III, two isolates to SCCmec type II. Most isolates were resistant to at least three of the non- $\beta$-lactam antibiotics tested. None of the strains exhibited resistance to new generation antibiotics, such as daptomycin and linezolid. Also, none of these strains showed resistance to tigecycline and only four strains were resistant to rifampin i.e. antibiotics which are very efficient in treating biofilm-associated infections.
\end{abstract}

Key words: Staphylococcus epidermidis, SCCmec type, antibiotic resistance

Among coagulase-negative staphylococci (CoNS), Staphylococcus epidermidis is the leading cause of hospital-acquired and biomaterial-associated infections. This bacterium can be responsible for endocarditis, peritonitis, bone and joint infections, septicaemia and bacteremia (Voung and Otto, 2002). The main virulence factor associated with S. epidermidis is the ability to form biofilm on implanted medical devices or damaged tissues. Strains belonging to this species have been particularly efficient at developing resistance to antimicrobial agents, which is due in part to the presence of the mobile genetic elements caring resistance genes (Schoenfelder et al., 2010). The mecA gene, which encodes PBP2a, a transpeptidase with a low affinity for beta-lactam antibiotics, is carried on a mobile genetic element called the staphylococcal chromosome mec (SCCmec). In addition, resistance gene for macrolides, tetracyclines and aminoglycosides can accrue on the SCCmec cassette. This element is bound by terminal inverted repeat sequences (IR) and integrated at the 3' end of the orf X gene, which is located near the origin of replication in the chromosome. Eleven types (I to XI) of SCCmec have been assigned for staphylococci based on the composition of the $c \mathrm{cr}$ gene com- plex and the class of the mec gene complex (Ito et al., 2001; 2004; IWG, 2009; Shore et al., 2013; Turlej et al., 2011). The mec gene complex is composed of mecA gene, intact or truncated sets of regulatory genes (mecRI and $m e c \mathrm{I}$ ), hypervariable region (HVR) and associated insertion sequence (IWG, 2009). The $c c r$ gene complex encodes the reccombinase that plays an important role in integration and excision of SCCmec from the chromosome. Three district $c c r$ genes, $c c r \mathrm{~A}, c c r \mathrm{~B}$ and $c c r \mathrm{C}$ have been described in staphylococci strains. In addition to the $c c r$ and mec gene complex, SCCmec cassette contains various mobile genetic elements (MGE), e.g. insertion sequences, transposons and plasmids, which are located in the joining regions (IWG, 2009). The occurrence of a very similar SCCmec cassette in different species provided evidence that genetic transfer of this element occurs between species in nature (Barbier et al., 2010; Hanssen and Ericson Sollid, 2006; Smyth et al., 2010). Moreover, Bloemendaal et al. (2010) demonstrated in vitro the transfer of SCCmec IV from S. epidermidis to the most virulent staphylococcal species, Staphylococcus aureus.

The aim of this study was to investigate the distribution of SCCmec types among S. epidermidis strains

* Corresponding author: E. Szczuka, Department of Microbiology, Institute of Experimental Biology, Faculty of Biology, Adam Mickiewicz University, Poznań, Poland; e-mail: ewasz@amu.edu.pl 
recovered from biomaterial-associated infections and analyse the antibiotic resistance patterns of these strains.

Sixty five CoNS collected from hospitalized patients were analyzed. Forty-six strains were isolated from the catheter-related bloodstream infection of hospitalized patients, which were regarded as causative agents of blood stream-infections (the same strain isolated from blood culture from the catheter and at the same time from blood culture by venous puncture). Nine strains were isolated from infection of the prosthesis, ten from peritoneal fluid. Isolates were identified by using the Vitek 2 system (bioMérieux, France).

The bacterial genomic DNA was isolated from clinical isolates using the Genomic DNA Plus kit (A\&A Biotechnology, Poland). The SCCmec types were identified using multiplex PCR (Zhang et al., 2005). The amplification products were electrophoresed in $1.5 \%$ agarose gel. The gels were stained with ethidium bromide, visualized on a UV light transilluminator, and documented with V.99 Bio-Print system (Vilber Lourmat, Torcy, France).

Resistance to $\beta$-lactams were determined by the cefoxitin $(30 \mu \mathrm{g})$ screen test. Susceptibility to the following antibiotical agents: fluoroquinolones (ciprofloxacin, levofloxacin, moxifloxacin, norfloxacin), aminoglycosides (gentamicin, tobramycin), glycopeptides (teicoplanin, vancomycin), macrolides and lincosamides (clindamycin, erythromycin), tetracyclines (tetracycline, tigecycline) and others (linezolid, rifampin, trimethoprim-sulfamethoxazole) was performed using Vitek 2 system (bioMérieux, France) according to EUCAST recommendations (http://www.eucast.org/clinical_breakpoints). Minimum inhibitory concentrations (MICs) of daptomycin were determined by microdilution method in Mueller-Hinton broth, supplemented to yield final concentration of $50 \mathrm{mg} / \mathrm{l}$ calcium (EUROCAST, 2007). Results were read after incubation at $37^{\circ} \mathrm{C}$ for $18-24 \mathrm{~h}$. Susceptibility to daptomycin was defined as $\mathrm{MIC}$ value of $\leq 1 \mathrm{mg} / \mathrm{l}$.

Tests were performed using software Excel (2010, Microsoft). The Pearson test was used to analyze correlation between SCCmec types and the resistance to different antibiotics. A P-value of $<0,05$ was considered significant.

We have previously documented the occurrence of biofilm-associated genes in the majority of clinical S. epidemidis as well as their ability to form biofilm structures in vitro (Szczuka and Kaznowski, 2014). This work aims to provide an insight into staphylococcal cassette chromosome mec elements and antibiotic resistance. Among these strains, $82 \%$ were multiresistant. Resistance to erythromycin, clindamycin, and tetracycline was found in 45 (69\%), 43 (66\%) and 35 (54\%) of the isolates, respectively. Twenty four $(37 \%)$ were resistant to ciprofloxacin, twenty one (32\%) to gentamicin and nineteen (29\%) to trimethoprim-sulfame- thoxazole. None of the strains exhibited resistance to glycopeptides. However, only four strains were resistant to rifampin and all were susceptible to tigecycline, antibiotics which are very effective in the treatment of biofilm-associated infections. All clinical strains were susceptible to linezolid, even though a few isolates of linezolid-resistant S. epidermidis were reported elsewhere (Hong et al., 2007; Treviño et al., 2009; Bonilla et al., 2010; Gu et al., 2013). In addition, all strains were susceptible to the new agent daptomycin, which demonstrated excellent in vitro activity against bacteria embedded in biofilms (Stewart et al., 2009).

It is believed that the increasing resistance of S. epidermidis to methicillin and other beta-lactam antibiotics is due to the presence of the SCCmec, which can be easily transferred between staphylococci strains, especially in biofilm structures (Garza-Gonzalez et al., 2010a; 2010b). Sixty two S. epidermidis isolates were classified into three SCCmec types. Forty five (69\%) S. epidermidis isolates harbour SCCmec type IV, which is believed to be the most mobile version of this element. Results of this study are in agreement with previously reported data, which indicated that type SCCmec type IV was the most prevalent in S. epidermidis strains among adults treated in a French hospital (Barbier et al., 2010; Garza-Gonzalez et al., 2010a; 2010b). Also SCCmec type IV dominated among S. epidermidis isolated from outpatients living in Algeria, Mali, Moldavia and Cambodia (Ruppé et al., 2009). In contrast, Li etal. (2009) found that only two out of 38 S. epidermidis strains recovered from patients treated in China carried SCCmec type IV, whereas SCCmec type III was the most prevalent. Our results indicate that 15 S. epidermidis strains (23\%) carried SCCmec type III. Only two isolates harboured SCCmec type II. None of the isolates carried type I. Our results demonstrated that the strains harbouring SCCmec cassette type III were in a significantly higher proportion resistant to non beta-lactam drugs, except rifampin as compared to isolates with SCCmec type IV (Table I). It could be explained by the presence of several resistance genes in the

Table I

Association between SCCmec types and resistance patterns to selected antimicrobial agents

\begin{tabular}{|c|c|c|c|}
\hline \multirow{2}{*}{$\begin{array}{l}\text { Antimicrobial } \\
\text { agents }\end{array}$} & \multicolumn{2}{|c|}{ Number of resistant isolates (\%) } & \multirow{2}{*}{ p-value } \\
\hline & $\begin{array}{l}\text { SCCmec type III } \\
(\mathrm{n}=15)\end{array}$ & $\begin{array}{c}\text { SCCmec type IV } \\
(\mathrm{n}=45)\end{array}$ & \\
\hline ciprofloxacin & $11(73)$ & $11(24)$ & $<0.001$ \\
\hline clindamycin & $13(86)$ & $27(60)$ & 0.031 \\
\hline gentamicin & $9(60)$ & $7(15)$ & $<0.001$ \\
\hline tetracycline & $14(93)$ & $16(35)$ & $<0.001$ \\
\hline $\begin{array}{l}\text { trimethoprim/ } \\
\text { ulfamethoxazole }\end{array}$ & $9(60)$ & $10(22)$ & $<0.001$ \\
\hline
\end{tabular}


SCCmec cassettes type III. The distribution of SCCmec types among $S$. epidermidis are comparable to the results of the studies conducted by Wisplinghoff et al. (2003), but different from the findings reported by Svensson et al. (2011). It has been reported that only three isolates carried known types of SCCmec - type III, whereas many strains contained multiple copies of $\mathrm{ccr}$ gene complexes and one class of mec gene complex. It is thought that the presence of different types of $\mathrm{ccr}$ complex in SCCmec elements might be due to rearrangements of types of SCCmec in bacterial cells (Hanssen et al., 2006). In our studies, only three strains could not be assigned to known SCCmec types because $c c r$ gene in these strains could not be determined. These strains contained mec complex B.

In conclusion, our results demonstrate the conservation of SCCmec element of S. epidermidis clinical isolates. These strains constitute a reservoir of SCCmec type IV. Although we found that the majority of S. epidermidis strains showed resistance to several antibiotics, no isolate showed resistance to daptomycin and tigecycline and only few isolates were resistant to rifampin which are the most efficient antibiotics against S. epidermidis biofilm-associated infections.

\section{Literature}

Barbier F., E. Ruppé, D. Hernandez, D. Lebeaux, P. Francois, B. Felix, A. Desprez, A. Maiga, P.L. Woerther, K. Gaillard and others. 2010. Methicillin-resistant coagulase-negative staphylococci in the community: high homology of SCCmec IVa between Staphylococcus epidermidis and major clones of methicillin-resistant Staphylococcus aureus. J. Infect. Dis. 202: 270-281.

Bloemendaal A.L., E.C. Brouwer and A.C. Fluit. 2010. Methicillin resistance transfer from Staphylocccus epidermidis to methicillinsusceptible Staphylococcus aureus in a patient during antibiotic therapy. PLoS One 5: e11841.

Bonilla H., M.D. Huband, J. Seidel, H. Schmidt, M. Lescoe, S.P. McCurdy, M.M. Lemmon, L.A. Brennan, A. Tait-Kamradt, L. Puzniak and others. 2010. Multicity outbreak of linezolid-resistant Staphylococcus epidermidis associated with clonal spread of a cfr-containing strain. Clin. Infect. Dis. 51: 796-800.

European Committee on Antimicrobial Susceptibility Testing (EUCAST). 2014. Breakpoint tables for interpretation of MICs and zone diameters Version 4.0. http://www.eucast.org/clinical_breakpoints/, 2014.01.01.

Garza-Gonzalez E., D. Lopez, C. Pezina, W. Muruet, V. BocanegraGarcia, I. Munoz, C. Ramirez and J.M Llaca-Diaz. 2010a. Diversity of staphylococcal cassette chromosome mec structures in coagulasenegative staphylococci and relationship to drug resistance. J. Med. Microbiol. 59: 323-329.

Garza-Gonzalez E., R. Morfin-Otero, J.M. Llaca-Diaz and E. Rodriguez-Noriega. 2010b. Staphylococcal cassette chromosome mec (SCCmec) in methicillin-resistant coagulase-negative staphylococci. A review and the experience in a tertiary-care setting. Epidemiol. Infect. 138: 645-654.

Gu B., T. Kelesidis, S. Tsiodras, J. Hindler and R.M. Humphries. 2013. The emerging problem of linezolid-resistant Staphylococcus. J. Antimicrob. Chemother. 68: 4-11.
Hanssen A.M. and J.U. Ericson Sollid. 2006. SCCmec in staphylococci: genes on the move. FEMS Immunol. Med. Microbiol. 46: 8-20. Hong T., X. Li, J. Wang, C. Sloan and C. Cicogna. 2007. Sequential linezolid-resistant Staphylococcus epidermidis isolates with G2576T mutation. J. Clin. Microbiol. 45: 3277-3280.

Ito T., Y. Katayama, K. Asada, N. Mori, K. Tsutsumimoto, C. Tiensasitorn and K. Hiramatsu. 2001. Structural comparison of three types of staphylococcal cassette chromosome mec integrated in the chromosome in methicillin-resistant Staphylococcus aureus. Antimicrob. Agents Chemother. 45: 1323-1336.

Ito T., X.X. Ma, F. Takeuchi, K. Okuma, H. Yuzawa and K. Hiramatsu. 2004. Novel type V staphylococcal cassette chromosome mec driven by a novel cassette chromosome recombinase, ccrC. Antimicrob. Agents Chemother. 48: 2637-2651.

International Working Group on the Classification of Staphylococcal Cassette Chromosome Elements (IWG). 2009. Classification of Staphylococcal Cassette Chromosome mec (SCCmec). Guidelines for Reporting Novel SCCmec Elements. Antimicrob. Agents Chemother. 53: 4961-4967.

Li M., X. Wang, Q. Gao and Y. Lu. 2009. Molecular characterization of Staphylococcus epidermidis strains isolated from a teaching hospital in Shanghai, China. J. Med. Microbiol. 58: 456-461.

Ruppé E., F. Barbier, Y. Mesli, A. Maiga, R. Cojocaru, M. Benkhalfat, S. Benchouk, H. Hassaine, I. Maiga, A. Diallo and others. 2009. Diversity of staphylococcal cassette chromosome mec structures in methicillin-resistant Staphylococcus epidermidis and Staphylococcus haemolyticus strains among outpatients from four countries. Antimicrob. Agents Chemother. 53: 442-449.

Schoenfelder S.M.K., K. Lange, M. Eckart, S. Hennig, S. Kozytska and W. Ziebuhr. 2010. Success through diversity - How Staphylococcus epidermidis establishes as a nosocomial pathogen. Int. J. Med. Microbiol. 300: 380-386.

Shore C. and D.C. Coleman. 2013. Staphylococcal cassette chromosome mec: recent advances and new insights. Int. J. Med. Microbiol. 303: 350-359.

Smyth D.S., A. Wong and D.A. Robinson. 2010. Cross-species spread of SCCmec IV sub-types in staphylococci. Infect. Genet. Evol. 11: 446-453.

Svensson K., B. Hellmark and B. Söderquist. 2011. Characterization of SCCmec elements in methicillin-resistant Staphylococcus epidermidis isolated from blood cultures from neonates during three decades. APMIS 119: 885-893.

Stewart P.S., W.M. Davison and J.N. Steenbergen. 2009. Daptomycin rapidly penetrates a Staphylococcus epidermidis biofilm. Antimicrob. Agents. Chemother. 53: 3505-3507.

Szczuka E. and A. Kaznowski. 2014. Antimicrobial activity of tigecycline alone or in combination with rifampin against Staphylococcus epidermidis in biofilm. Folia Microbiol. 59: 283-288.

Treviño M., L. Martínez-Lamas, P.A. Romero-Jung, J.M. Giráldez, J. Alvarez-Escudero and B.J. Regueiro. 2009. Endemic linezolidresistant Staphylococcus epidermidis in a critical care unit. Eur. J. Clin. Microbiol. Infect. Dis. 28: 527-33.

Turlej A., W. Hryniewicz and J. Empel. 2011. Staphylococcal Cassette Chromosome mec (SCCmec) classification and typing methods: an Overview. Pol. J. Microbiol. 60: 95-103.

Wisplinghoff H., A.E. Rosato, M.C. Enright, M. Noto, W. Craig, and G.L. Archer. 2003. Related clones containing SCCmec type IV predominate among clinically significant Staphylococcus epidermidis isolates. Antimicrob. Agents Chemother. 47: 3574-3579.

Voung C. and M. Otto. 2002. Staphylococcus epidermidis infections. Microb. Infect. 4: 481-489.

Zhang K., J. McClure, S. Elsayed, T. Louie and J.M. Clony. 2005. Novel PCR Assay for characterization and concomitant subtyping of staphylococcal cassette chromosome mec Types I to V in Methicillin-

Resistant Staphylococcus aureus. J. Clin. Microbiol. 43: 5026-5033. 
